\title{
summary-Cochrane Review
}

\section{Fluoride gel applications reduce caries incidence}

\author{
Marihno VCC, Higgins JPT, Logan S, Sheiham A. Fluoride gels for preventing dental caries in children and adolescents. \\ (Cochrane review). In: The Cochrane Library, Issue 2, 2002. Oxford: Update Software
}

Question: Do fluoride gels reduce dental caries?

Objective: To determine the effectiveness and safety of fluoride gels in the prevention of dental caries in children and to determine the relationship to initial caries severity, background exposure to fluoride, and the mode and frequency of gel use.

Data Sources: The purpose was to identify all relevant studies from 1965. Electronic searching of the MEDLINE, EMBASE, SCISEARCH, SSCISEARCH, ISTP, BIOSIS, CINAHL, ERIC, Dissertation Abstracts, LILACS/BBO databases and of the Cochrane Controlled Trial and Medline registers has been attempted. Furthermore, all eligible trials, meta-analyses and review articles were scanned for relevant references. In addition 7 journals were hand searched and personal contact with authors and manufacturers was achieved while searching for unpublished data.

Study Selection: Exclusively RCTs and quasi-RCTs with blind outcome assessments, comparing groups applying fluoride gel with placebo or no treatment groups consisted of children up to 16 years old at baseline were included in this review. Studies were excluded where the participants were selected on the basis of special health conditions, were carrying orthodontic bands (or other appliances), and/or used additional caries preventive agents (eg chlorhexidine or sealants etc.), as well as studies that provided results only on plaque/ gingivitis, calculus, dentin hypersensitivity or physiological fluoride.

Data Extraction and Synthesis: The first reviewer assessed the quality of all included studies. The second reviewer duplicated the process for a random sample of $\approx 1 / 3$ of them. In addition, the 2 nd reviewer independently assessed any study that could not be classified by the first. A third reviewer was consulted to resolve any disagreement. Attempts were made to contact authors of trials that could not be classified in order to ascertain whether the inclusion criteria were met. Agreement was good for allocation (kappa=0.61) and blinding (kappa=0.73). There was a considerable difference in the quality of the 25 studies in this review. Random effects meta-analyses were performed where data could be pooled. Potential sources of heterogeneity were examined in random effects meta-regression analyses.

Results: Twenty-five studies were included, involving 7747 children. For all the 23 trials, that contributed data for meta-analyses, combined the DMFS pooled prevented fraction estimate was 0.28 , suggesting a substantial benefit from the use of fluoride gel.

Statistically significant heterogeneity was observed $(p<0.0001)$. Univariate meta regression analysis suggested no significant association between estimates of DMFS prevented fractions and length of followup, allocation concealment, blinding of outcome assessment or drop out rate. Populations with a caries incidence of 2.2 DMFS/year had an $\mathrm{NNT}=2(95 \% \mathrm{CI}=1$ to 3$)$. For populations with a caries incidence of $0.2 \mathrm{DMFS} / \mathrm{yr}$ had an $\mathrm{NNT}=24(95 \% \mathrm{CI}=18$ to 36$)$. A funnel plot suggests a balancing asymmetry with a single large study demonstrating the largest positive effect and a single small study demonstrating a large deleterious effect. Only two studies reported on adverse effects (nausea/vomiting).

Conclusions: This review suggests that the application of fluoride gels, either professionally or self-applied, is associated with a substantial reduction in caries increment. This effect was independent of other fluoride sources, but did depend on application frequency. Given the large beneficial effect, further demonstration of efficacy is not needed. However, it is important that future trials examine potential adverse effects as well as application frequency.

Evidence-Based Dentistry (2002) 3, 64-65. doi:10.1038/ sj.ebd.6400133

Note: Abstracts of all Cochrane Reviews are available to download for free at http://www.update-software.com/cochrane/abstract.htm

\section{Commentary}

The use of topical fluoride treatment for caries prevention is a frequent and increasing practice. Surveys conducted by the American Dental Association ${ }^{1}$ (ADA) show that the proportion of all patients receiving fluoride treatments from private practitioners increased from $4 \%$ in 1969 to $10.6 \%$ in 1999 . It is estimated that $70 \%$ of children in
Canada who make an annual dental visit receive a topical fluoride treatment ${ }^{2}$. Because of the changing epidemiology of the distribution of dental caries, the US Centers for Disease Control and Prevention ${ }^{3}$ and the $\mathrm{ADA}^{4}$ recommend topical fluoride treatment only for high risk children. Many dentists are not following these recommendations. A study of more than 15000 insured children in Michigan showed that two-thirds were receiving fluoride treatments at every recall visit, by dentists who provide this service at least $98 \%$ of the time with each recall examination, without selectivity $^{5}$. Against this backdrop of use, change in disease prevalence, potential adverse effects, and the availability of other caries preventive modalities, sev- 
eral fundamental clinical questions are apparent. 1) Should fluoride gel be used at all? 2) If used, what is the appropriate fluoride compound concentration, frequency, duration, delivery system and method of application? 3) Are the preventive effects from fluoride gel in addition to fluoride dentifrice and/or fluoridated water of clinical significance? 4) Should fluoride gel be used instead of or in addition to other caries preventive agents? 5) Should it be provided to all children or should selection criteria be used based on the caries suceptibility of the individual or the caries prevalence in the community? So, should fluoride gel be used, and if so, how? The results of this comprehensive, well-conducted systematic review and meta-analysis provide answers to some of these questions. There is strong evidence, based on numerous randomized clinical trials, to support its effectiveness in preventing dental caries among school-age children, based on pooled data from 23 studies. Interestingly, the authors evaluated the effects of baseline DMFS levels, exposure to other fluoride sources, professional or self-application, tray or brush application, application frequency, concentration and intensity, and aspects of the study design. There was much heterogeneity among the studies with respect to these factors. However, with the exception of the very first study in $1967^{6}$, none of these variables were significantly associated with the DMFS. Thus based on this systematic review, the effectiveness of the gel may not vary greatly due to differences in any of these factors. The choice of whether to use fluoride gel will need to be made based on other factors such as cost, convenience and patient preference.

This systematic review comes to a similar conclusion as a 1998 systematic review by van Rijkom ${ }^{7}$ and colleagues, yet shares only 14 studies in common with the current review. Van Rijkom's analysis also evaluated baseline caries prevalence, background fluoride exposure, concentration, tray or brush method, and application frequency, and similarly found no significant effect for any of these factors.

There remain a number of questions. Neither analysis provided information about the gel's effectiveness in the primary dentition, among adults or in patients with special needs. Neither analysis included studies directly comparing different caries preventive methods or combinations of methods. Neither of these meta-analyses addressed the issue of using caries risk selection criteria. However, one might extrapolate from the data provided. The number needed to treat (NNT) varies inversely as the DMFS. This indicates that fluoride gel is much more effective in patients with a high incidence than patients with a low incidence. Thus one might ask whether patients with a low incidence are being over medicated.

Authors of a third recent systematic review of topical fluoride methods of caries prevention in high risk individuals rated the evidence as insufficient (except for fluoride varnish) because of too few studies ${ }^{8}$. However, this third systematic review used much more stringent inclusion criteria, and thus included far fewer studies. Finally, the Cochrane Collaboration has protocols on file to address some of these issues, so perhaps additional answers will be forthcoming.

1. American Dental Association. Future of Dentistry. Chicago: American Dental Association, Health Policy Resources Center: 2001.

2. Johnston DW, Lewis DW. Three-year randomized trial of professionally applied topical fluoride gel comparing annual and biannual applications with/without prior prophylaxis. Caries Res 1995; 29:331-336.

3. Centers for Disease Control and Prevention. Recommendations for using fluoride to prevent and control dental caries in the United States. MMWR 2001; 50(No.RR-14): 18, 21.

4. American Dental Association Ad hoc Committee on Caries Prevention. Caries diagnosis and risk assessment. JADA 1995; (Spec Suppl):S1-S24.

5. Eklund SA, Pittman JL, Heller KE. Professionally applied topical fluoride and restorative care in insured children. J Public Health Dent 2000; 60:33-38.

6. Englander $H R$, Keyes $P H$, Gestwicki $M$, Sultz HA. Clinical anticaries effect of repeated topical sodium fluoride applications by mouthpieces. JADA 1967; 75:638-644.

7. Van Rijkom HM, Truin GJ, van't Hof MA. A meta-analysis of clinical studies on the caries-inhibiting effect of fluoride gel treatment. Caries Res 1998; 32:83-92.

8. Bader JD, Shugars DA, Bonito AJ. Systematic reviews of selected dental caries diagnostic and management methods. J Dent Ed 2001; 65:960-968.

Jane A Weintraub Division of Oral Epidemiology and Dental Public Health, University of California, San Francisco, USA 